\section{\$26. Simple Core-SOL-Divertor Model to Investigate Plasma Operational Space}

Hiwatari, R. (CRIEPI: Central Research Institute of Electric Power Industry), Kuzuyama, Y. (Keio Univ.), Hatayama, A. (Keio Univ.), Okano, K. (CRIEPI), Asaoka, Y. (CRIEPI), Zhu, S. (Institute of Plasma Physics, China), Tomita, Y.

Consistency of the edge plasma operation with the core plasma operation is an important issue for the design of ITER and the future fusion power plant. In case of the ITER divertor modeling, the fitting scaling laws for divertor plasma property are built with a two dimensional (2D) divertor transport code, and these are used as boundary conditions for the core plasma analysis.1) Being prior to such detailed and massive calculations by multi-dimensional transport codes, it is necessary to understand qualitative and overall features of the plasma operational space including the requirements for the SOL and divertor plasma. For this purpose, we are developing a simple Core-SOL-Divertor (C-S-D) model.2,3)

The transport models applied to the C-S-D model are a $0 \mathrm{D}$ core plasma model based on ITER physics guidelines and a usual two-point model for SOL-divertor region. The key issue of this C-S-D model is how to combine the two-point model with the $0 \mathrm{D}$ core plasma model. Usually, the upstream SOL density is a given parameter in the two-point model. In the C-S-D model, the particle balance for SOL-divertor region including the neutral transport is solved to evaluate the upstream SOL density $n_{s}$. We assume that all neutral particles are originated at the divertor plate. Its generation rate is supposed to be proportional to the particle flux to the divertor plate. Consequently, the following definition of the total neutral source rate $N_{\mathrm{n}}$ including gas puff $N_{\text {puif }}$ at the edge region is applied;

$$
N_{\mathrm{n}}=n_{\mathrm{d}} M_{\mathrm{d}} C_{\mathrm{s}} 2 \pi R h \Delta_{\mathrm{n}} \sin (\psi)+N_{\text {puff }},
$$

where $n_{\mathrm{d}}, M_{\mathrm{d}}, C_{\mathrm{s}}, R$ and $\psi$ are density, mach number and sound velocity, major radius and the angle of the magnetic field at the divertor plate, respectively. The density decay length $\Delta_{n}$ is assumed to be twice of temperature decay length of SOL. By using this simple neutral model and the particle flux across the separatrix $\Gamma_{\text {core }}$ from the $0 D$ core plasma calculation, the particle balance equation for the SOL-divertor region becomes

$$
\Gamma_{\text {core }} S_{\text {cure }}+N_{\mathrm{n}}^{\mathrm{sol}}+N_{\mathrm{n}}^{\mathrm{div}}=n_{\mathrm{d}} M_{\mathrm{dl}} C_{\mathrm{s}} \sin (\psi) S_{\text {div }}
$$

where $N_{n}^{\text {sol }}\left(N_{n}^{\text {div }}\right)$ is the total number of ionized particles in the SOL (divertor) region, and $S_{\text {core }}$ and $S_{\text {div }}$ are the areas for the core and divertor region normal to the particle flux. The ionized particle numbers are defined by the ionization fraction in the SOL and the divertor region $\left(f_{\text {ion }}^{\text {sol }}\right.$ and $f_{\text {ion }}^{\text {div }}$ ). Their definitions are
$N_{\mathrm{n}}^{\mathrm{sol}}=f_{\text {ion }}^{\mathrm{sol}}\left(1-f_{\mathrm{ion}}^{\mathrm{div}}\right) N_{\mathrm{n}}$ and $N_{\mathrm{n}}^{\mathrm{div}}=f_{\text {ion }}^{\mathrm{div}} N_{\mathrm{n}}$. The ionization fraction in the divertor region is modeled by $f_{\text {ion }}^{\text {div }}=1-\exp \left(-\left(L_{\mathrm{d}} \sin (\psi)\right) / \lambda_{\text {ion }}^{\text {div }}\right)$, where $L_{\mathrm{d}}$ and $\lambda_{\text {ion }}^{\text {div }}$ are the length of the divertor region and the mean free path of the neutral particle, respectively. The ionization fraction in the SOL region is defined by $f_{\text {ion }}^{\text {sol }}=A_{\text {sol }}\left(A_{\text {core }}+A_{\text {sol }}+A_{\text {pump }}\right)$, where $A \mathrm{j}$ denotes the effective area for core plasma, $\mathrm{SOL}$ and pumping effect, respectively.

To check the validity of this C-S-D model, comparison with the edge transport code (B2-EIRENE) is carried out. We focus on the JT-60U L-mode discharge in the high recycling state 4), and it is shown that the result by the C-S-D model is reasonable qualitatively and quantitatively, however, other validation for the neutral transport should be carried out because of the simple neutral model.

The C-S-D model is applied to the LH transition phase of ITER. The LH transition condition is modeled by using the scaling law of LH transition threshold power. The time evolution of SOL-divertor parameters is shown in Fig. 1. The particle confinement time of core plasma is calibrated so as to reproduce the ITER reference upstream SOL density. Fig. 1 reveals that there is a possibility of divertor density $\left(n_{\mathrm{d}}\right)$ oscillation, while the core density (which is not shown in Fig. 1) and the upstream SOL density increase. Such $n_{\mathrm{d}}$ oscillation has an effect on the divertor operation in the ITER and the future fusion reactor, and should be examined more carefully.

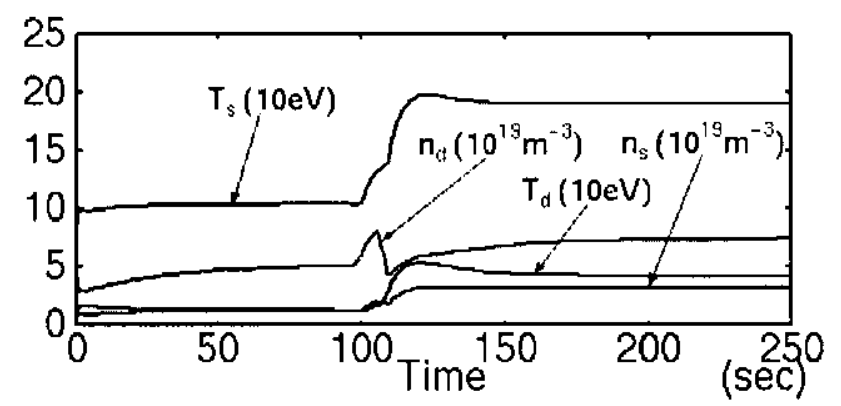

Fig. 1. Time evolution of the SOL-divertor parameters (density and temperature) by the C-S-D model. The subscripts $\mathrm{s}$ and $\mathrm{d}$ correspond to the upstream $\mathrm{SOL}$ and the divertor, respectively.

As shown in Fig.1, this C-S-D model is useful to investigate consistent core and edge plasma operational space. By the C-S-D model, the steady state operational space of HT-7U is under investigation[3]. This work is partly supported by JSPS-CAS Core-University Program on Plasma and Nuclear Fusion.

\section{Reference}

1) Pacher, G.W. et al.: Nucl. Fusion 43 (2003) 188

2) Hiwatari, R. et al: Contrib. Plasma Phys. 44 (2004) 76

3) Hiwatari, R. et al.: "Simple Core-SOL-Divertor Model and its Application to HT7U", $16^{\text {th }}$ International Conference on Plasma Surface Interactions in Controlled Fusion Devices, Portland USA, May 24-28 2004

4) Hatayama, A. et al: Nucl. Fusion $40(2000) 2009$ 